\title{
New Treatment New Complication - Mini Review
}

\author{
Ákos Géza Pethő* \\ Semmelweis University, Faculty of Medicine 1083 Budapest, Hungary
}

*Corresponding author: Ákos Géza Pethő, Department of Internal Medicine and Oncology, Semmelweis University, Faculty of Medicine 1083 Budapest, KorányiSándor u. 2/a. (Mailing address: 1428 Budapest, Pf. 2.), Hungary.

Received Date: May 15, 2020

Published Date: June 29, 2020

\begin{abstract}
Nephrotoxicity caused by medication could limit the treatment success. In multiple malignancies are playing crucial role the anti-cancer drugs which have well known nephrotoxicity. Using the conventional chemotherapies acute kidney injury could develop but not in all patients who are suffering cancers. In these patients will acute tubulointerstitial nephritis or rarely nephrotic syndrome occur. The onco-pharmacology is an intensively investigated field. In the recent decade's novel therapies founded. The new treatments in oncology have more targeted affect on cancer cells. These drugs will be modifying the whole immune system with the fine balance between cancer surveillance and preserving self-tolerance. The immune checkpoint inhibitors have superior anti-cancer therapeutic affect but new, until unknown complication raised. In this mini review we will discuss the most acute kidney injuries during chemotherapy.
\end{abstract}

Keywords: Cancer; Chemotherapy; Immune system; Immune checkpoint inhibitors

\section{Introduction}

Nephrotoxicity caused by medication could limit the treatment success. In malignancies the conventional chemotherapeutic medications could cause acute or chronic kidney injury. The mechanism of the kidney injury is nontargeted cell killing with the possibility of the injure the renal microvasculature, glomerulus, tubular segments, and renal interstitial [1]. The clinical syndrome that caused by those conventional chemotherapeutic drugs could be various, e.g. acute kidney injury, proteinuria-hematuria, the nephrotic syndrome, isolated tubulopathies (with accompanying electrolyte and acid-base disturbances), hypertension, and chronic kidney disease [2]. Beyond these conventional chemotherapeutic medications novel cell specific immunotherapies were developed. Oncologists have used immunotherapies since the 1980s and 1990s. The first immunotherapies used were exogenous cytokines, such as IFN- $\alpha$ [3]. From this point intensively research was started, the anti-cancer therapies will have advanced further by intensifying the immune response [4]. The immunotherapies will fine balance between cancer surveillance and preserving self-tolerance. This novel immunotherapy known as immune checkpoints inhibitors. The modulation of the immune system is dependent on the complex interplay between multiple immune system components. In the pathway of the immune system respond playing rolecytotoxic, helper, and regulatory T cells; macrophages; natural killer cells; and myeloid-derived suppressor cells. By the respond of thy immune system is the most important point; the balance between a hyperactive immune response resulting in immune-mediated damage to healthy tissues and a hypoactive immune response resulting in infections and malignancies is achieved through a redundant and multilevel regulation of lymphocyte cytotoxic activity through immune checkpoint inhibition [5]. The novel immunotherapies have new side effects. Off-target inflammatory responses to checkpoint inhibitors are commonly referred to as immune-related adverse effects (irAEs).

\section{Conventional Chemotherapies}

Not all patients exposed to nephrotoxic chemotherapeutic agents develop kidney injury, suggesting the presence of several 
factors that enhance patient risk for nephrotoxicity. Most common is acute tubular injury or necrosis due to treatment with platinumcontaining regimens, ifosfamide, oledronic acid, pemetrexed, and numerous other chemotherapeutic agents [1]. But not only the renal tubulointerstitial and well known tubulopathy could occur. Some drugs could affect intraglomerular, causing focal and segmental glomerulosclerosis and minimal change disease, which promotes a form of drug-induced podocytopathy. ss such as pamidronate are widely used in the treatment of patients with lytic bony lesions secondary to breast cancer or multiple myeloma. Pamidronate causes a collapsing variety of FSGS [6]. Bisphosphonates have been associated with deterioration of renal function and histopathological changes in the kidney. Drug-related side effects are limiting factors to the use of bisphosphonates. Available data suggest that pamidronate and zolidronate, but not ibandronate, are associated with nephrotoxicity in the treatment of patients with malignant disease [7]. Other drug-induced forms of acute kidney injury include obstructive and inflammatory interstitial injury resulting from intratubular crystal precipitation induced by methotrexate and interstitial nephritis from various chemotherapeutic agents [8].

\section{IFN- $\alpha$ Therapy}

IFN- $\alpha$ was Food and Drug Administration (FDA) approved to treat CML in 1981 followed by hairy cell leukemia, AIDS-related Kaposi sarcoma, metastatic melanoma, and follicular non-Hodgkin lymphoma. IFN- $\alpha$ will enhances the effect or $\mathrm{T}$ cell-mediated responses, with cytokine release, e.g. IL-12 secretion, via several signaling events [9]. By IFN- $\alpha$ treatment could occur minimal change disease (MCD) or FSGS, which are manifestations of podocyte injury. Sometimes thrombotic microangiopathy (TMA) is the clinical symptom after administering of IFN- $\alpha$, which reflects vascular endothelial damage [10].

\section{Immune Checkpoint Inhibitors}

The immune checkpoint inhibitors are more interesting field of anti-cancer drugs. Enhancing or suppressing $\mathrm{T}$ cell activation via costimulatory or coinhibitorymolecules modifies effector $\mathrm{T}$ cell response. Cytotoxic lymphocyte-associated antigen-4 (CTLA4) and programmed cell death protein-1 (PD-1) are two receptors that play an important role in negatively regulating $\mathrm{T}$ cell activation and function [11]. The most important effect of those receptors. that ligand binding to CTLA- 4 and PD- 1 receptors modifies the immune system response to antigens by inhibiting $\mathrm{T}$ cell activation. The inhibition of the T-cells allows the immunologic self-tolerance and prevents autoimmunity. Administration of immune checkpoint inhibitors could various of adverse autoimmune effects appear, e.g. dermatitis, colitis, pneumonitis, endocrinopathies. The incidence of IRAEs range from $15 \%$ to $90 \%$, with severe IRAEsranging from $0.5 \%$ to $13 \%[12,13]$. The mechanism of CPI-induced kidney injury is unknown, but CPI therapy in patients with kidney transplants could develop rejection. Because of this major side effect of CPI oncologist should consider the anti-cancer therapy in kidney transplanted patients [14]. The anti-CTLA4 drug (ipilimumab) in some cases caused minimal-change disease and interstitial nephritis. The ipilimumab has been described as causing acute kidney injury from interstitial nephritis as well as lupus nephritis, too [15]. Pathology revealed acute interstitial nephritis in most cases with varying degrees of foot process effacement. Most of the AKI occurred 6-12 weeks following the start of treatment, with the longest interval being 26 weeks [16]. Programmed cell death protein 1 (PD-1) play a crucial role in anti-cancer immunity, too. Monoclonal antibodies against PD-1 (nivolumab and pembrolizumab) andPD-L1 (atezolizumab) are currently used for the treatment of advanced stage cancers. Acute tubulointerstitial nephritis is an important manifestation of kidney injury associated with the use of anti-PD-1 drugs [17]. Nivolumab, a monoclonal anti-PD1 antibody can cause autoimmune glomerulonephritis as well as tubulointerstitial injury. The immune-related nephritis generally responded well to systemic corticosteroid treatment [18]. The oncologist almost uses combination therapy with ipilimumab and nivolumab. The combination therapy appears to increase the incidence and severity of adverse events as compared to the use of nivolumab alone [19].

\section{Conclusion}

The acute kidney injury is the most complication of the anticancer chemotherapy. The conventional anti-cancer drugs have dose effect and the possibility of the kidney injury raises with the decrease of the kidney function. In most cases after administrating the conventional chemotherapies acute tubulointerstitial nephritis will develop. The oncology treatment has novel drugs, which has more targeted anti-cancer effect. These new therapies are modifying the immune system. With the modification of the immune system will develop new symptoms and side effects. All potential causes of AKI (prerenal, ATN, obstruction) should be evaluated. If AKI is confirmed on a kidney biopsy as AIN or a podocytopathy. Strongly recommended the discontinuation of the check point inhibitors and a course of corticosteroids [20]. Empirical corticosteroid treatment may mask the progression of glomerular disease. However, biopsyconfirmed acute tubular necrosis does not require discontinuation of checkpoint inhibitor therapy, and while corticosteroid treatment appears to be harmless as far as oncologic outcomes are concerned, even a short-term course is associated with multiple systemic adverse effects [5]. The life expectancy of cancer patients is increasing, leading to a higher likelihood of developing some degree of kidney impairment and patients with kidney impairment are living longer, with an increased risk of developing concomitant cancer [21].

\section{Acknowledgement}

None

\section{Conflict of Interest}

None. 


\section{References}

1. Perazella MA (2012) Onco-nephrology: renal toxicities of chemotherapeutic agents. Clin J Am Soc Nephrol 7(10): 1713-1721.

2. Lameire N, Kruse V, Rottey S (2011) Nephrotoxicity of anticancer drugs-an underestimated problem. Acta Clin Belg 66(5): 337-345.

3. Belardelli F, Ferrantini M, Proietti E, Kirkwood JM (2002) Interferonalpha in tumor immunity and immunotherapy. Cytokine \& Growth Factor Reviews 13(2): 119-134.

4. Takeda K, Kojima Y, Uno T, Hayakawa Y, Teng MW, et al. (2010) Combination therapy of established tumors by antibodies targeting immune activating and suppressing molecules. J Immunol 184(10): 5493-5501.

5. Shingarev R, Glezerman IG (2019) Kidney Complications of Immune Checkpoint Inhibitors: A Review. American journal of kidney diseases 74(4): 529-537.

6. Shreedhara M, Fenves AZ, Benavides D, Stone MJ (2007) Reversibility of pamidronate-associated glomerulosclerosis. Proc (Bayl Univ Med Cent) 20(3): 249-253.

7. Jia N, Cormack FC, Xie B, Shiue Z, Najafian B, et al. (2015) Collapsing foca segmental glomerulosclerosis following long-term treatment with oral ibandronate: case report and review of literature. BMC Cancer 15: 535.

8. Perazella MA, Moeckel GW (2010) Nephrotoxicity from chemotherapeutic agents: clinical manifestations, pathobiology, and prevention/therapy. Semin Nephrol 30(6): 570-581.

9. Lesinski GB, Anghelina M, Zimmerer J, Bakalakos T, Badgwell B, et al (2003) The antitumor effects of IFN- $\alpha$ are abrogated in a STAT1-deficient mouse. Journal of Clinical Investigation 112(2): 170-180.

10. Kundra A, Wang JC (2017) Interferon induced thrombotic microangiopathy (TMA): Analysis and concise review. Crit Rev Oncol Hematol 112: 103-112.

11. Murakami N, Motwani S, Riella LV (2017) Renal complications of immune checkpoint blockade. Curr Probl Cancer 41(2): 100-110.
12. Champiat S, Lambotte O, Barreau E, Belkhir R, Berdelou A, et al. (2016) Management of immune checkpoint blockade dysimmune toxicities: a collaborative position paper. Ann Oncol 27(4): 559-574.

13. Cortazar FB, Marrone KA, Troxell ML, Ralto KM, Hoenig MP, et al. (2016) Clinicopathological features of acute kidney injury associated with immune checkpoint inhibitors. Kidney Int 90(3): 638-47.

14. Perazella MA, Shirali AC (2018) Nephrotoxicity of Cancer Immunotherapies: Past, Present and Future. J Am Soc Nephrol 29(8): 2039-2052.

15. Kidd JM, Gizaw AB (2016) Ipilimumab-associated minimal-change disease. Kidney Int 89(3): 720.

16. Fadel F, El Karoui K, Knebelmann B (2009) Anti-CTLA4 antibodyinduced lupus nephritis. N Engl J Med 361(2): 211-212.

17. Koda R, Watanabe H, Tsuchida M, Iino N, Suzuki K, et al. (2018) Immune checkpoint inhibitor (nivolumab)-associated kidney injury and the importance of recognizing concomitant medications known to cause acute tubulointerstitial nephritis: a case report. BMC Nephrol 19(1): 48

18.Jung K, Zeng X, Bilusic M (2016) Nivolumab-associated acute glomerulonephritis: a case report and literature review. BMC Nephrol 17(1): 188.

19. Postow MA, Chesney J, Pavlick AC, Robert C, Grossmann K, et al. (2015) Nivolumab and ipilimumab versus ipilimumab in untreated melanoma. N Engl J Med 372(21): 2006-2017.

20. Wanchoo R, Karam S, Uppal NN, Barta VS, Deray G, et al. (2017) Adverse Renal Effects of Immune Checkpoint Inhibitors: A Narrative Review. Am J Nephrol 45(2): 160-169.

21. Silvestris N, Argentiero A, Cosmai L, Porta C, Gesualdo L, et al. (2019) Management of targeted therapies in cancer patients with chronic kidney disease, or on haemodialysis: An Associazione Italiana di Oncologia Medica (AIOM)/Societa' Italiana di Nefrologia (SIN) multidisciplinary consensus position paper. Crit Rev Oncol Hematol 140: 39-51. 